\title{
Stressed out hearts
}

Imatinib mesylate (Glivec) potently inhibits the kinase activity of the oncogenic fusion protein $\mathrm{BCR}-\mathrm{ABL}$ and is an effective treatment for chronic myeloid leukaemia. Although imatinib seems to be well tolerated by most patients, more than $60 \%$ of patients on imatinib in clinical trials develop peripheral oedema, a possible sign of cardiotoxicity. However, heart function was not assessed in any of the clinical trials with this agent. Thomas Force and colleagues report that 10 patients who previously had normal heart function developed severe heart failure after the initiation of imatinib therapy. They found that imatinib is not directly toxic, but that the inhibition of one of its targets, $\mathrm{ABL}$, triggers the stress response in cardiomyocytes and induces cell death.

Myocardial biopsy samples taken from patients showed prominent membrane whorls in the myocytes, an abnormality that is a characteristic of toxin-induced myopathies. The cells also showed pleiomorphic mitochondria and dilated endoplasmic reticulum (ER), which are signs of stress.

To investigate this further, the authors treated healthy mice with imatinib and saw similar structural changes in the mouse hearts. Doses of imatinib given to produce blood concentrations comparable to those in humans led to left ventricular dysfunction. Imatinib seemed to cause necrotic cell death (as seen by the dose-dependent collapse of mitochondrial membrane potential, release of cytochrome $c$ and pronounced cytosolic vacuolization) rather than apoptosis. Gene transfer of an imatinib-resistant mutant of ABL inhibited the imatinib-induced release of cytochrome $c$ and protected from cell death. This suggests that the inhibition of ABL by imatinib is the mechanism of cardiomyocyte toxicity.

What are the mechanisms that regulate imatinib-induced cardiomyocyte death? Because of the observed ER dilation, Force and colleagues investigated the ER stress-response pathway and found that imatinib activated both the EIF2 $\alpha$ and IRE1 parts of this pathway in imatinib-treated mice. Although this response is initially protective, if the inducing stress to cells is not relieved - as occurs with prolonged imatinib therapy - IRE1 can signal cell death by activating the pro-death JNK pathway. The authors found that JNK was activated in the hearts of imatinib-treated mice and that this activation was reduced by treatment with salubrinal, a small-molecule inhibitor of EIF2 $\alpha$ dephosphorylation. Furthermore, the inhibition of either EIF2 $\alpha$ dephosphorylation with salubrinal or JNK activity with a peptide inhibitor rendered cardiomyocytes resistant to the imatinibinduced collapse of mitochondrial

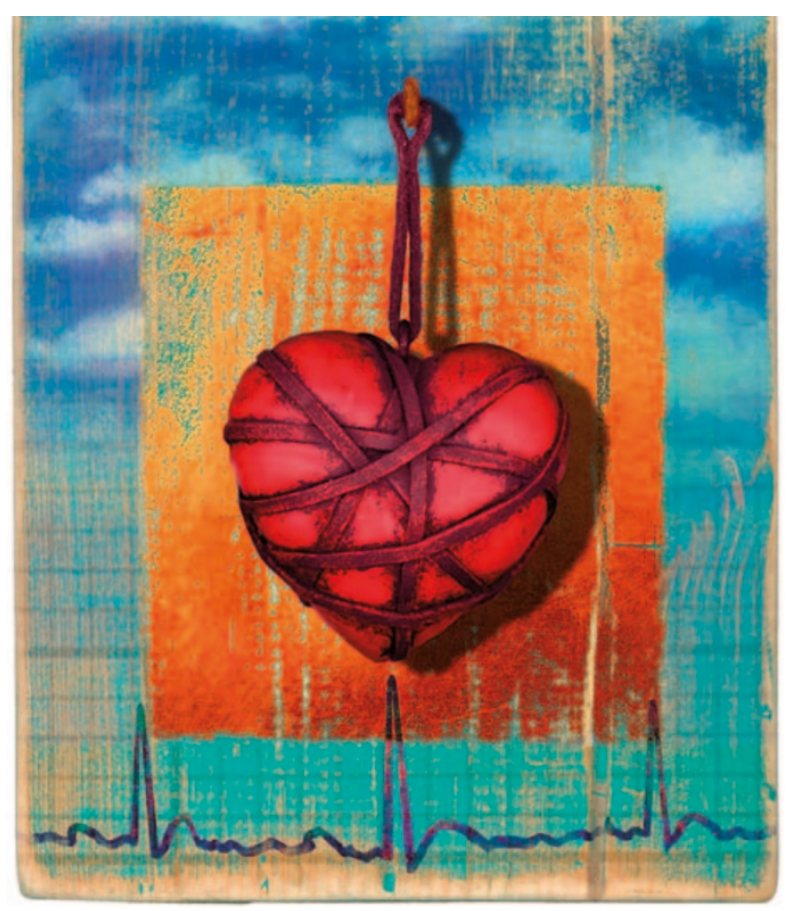

membrane potential and cell death. Therefore, the imatinib-mediated induction of the ER stress-response pathway leads to cell death through the activation of JNKs.

These findings not only suggest that patients who are on imatinib should be monitored closely for signs of left ventricular dysfunction, but also that clinical trials of new agents that target $\mathrm{ABL}$ should prospectively assess left ventricular function so that rates of cardiotoxicity can be determined.

Ezzie Hutchinson ...patients who are on imatinib should be monitored closely for signs of left ventricular dysfunction
ORIGINAL RESEARCH PAPER Kerkelä, R. et al

Cardiotoxicity of the cancer therapeutic agent imatinib mesylate. Nature Med. 23 July 2006 (doi: 10.1038/nm1446)

\section{RESEARCH HIGHLIGHTS ADVISORS}

AVI ASHKENAZI Genentech, Inc., South San Francisco, CA, USA JOSE BASELGA

Vall d'Hebron University Hospital,

Barcelona, Spain

ANTON BERNS

Netherlands Cancer Institute,

Amsterdam, The Netherlands
MARIA BLASCO

Spanish National Cancer Centre (CNIO), Madrid, Spain RON DEPINHO Harvard Medical School, Boston, MA, USA GLENN DRANOFF

Dana-Farber Cancer Institute, Boston, MA, USA
RAKESH JAIN

Massachusetts General Hospital, Boston, MA, USA CHRISTOPH LENGAUER Novartis Institute for Biomedical Research Inc., Cambridge, MA, USA LANCE LIOTTA National Cancer Institute, Bethesda, MD, USA
JOHN D. POTTER

Fred Hutchinson Cancer Research Center, Seattle, WA, USA

DAVID SIDRANSKY

Johns Hopkins University

School of Medicine, Baltimore, MD, USA
BERT VOGELSTEIN

The Sidney Kimmel Comprehensive Cancer Center, Baltimore, MD, USA ROBERT WEINBERG

Whitehead Institute for Biomedical Research, Cambridge, MA, USA ZENA WERB University of California at San Francisco, CA, USA 


\section{In the news}

\section{DRUG'S CANCER LEGACY}

The dangers of diethylstilbestrol (DES), a synthetic oestrogen-like hormone given to pregnant women who are at risk of miscarriage, became evident when the daughters of mothers who received the drug developed rare cancers of the cervix and vagina. Exposed mothers were more likely to develop breast cancer, but it was not clear whether their daughters were similarly affected.

A new US study examined the occurrence of breast cancer in a cohort of 4,817 so-called 'DES daughters' compared with 2,073 unexposed women. The key finding, reported in the August issue of Cancer Epidemiology, Biomarkers \& Prevention, was that the risk of developing breast cancer was 1.9 times greater in women over the age of 40 who had been exposed to DES in the womb.

According to Julie Palmer, professor of epidemiology at the Boston University School of Public Health and lead researcher on the study: "This is really unwelcome news because so many women worldwide were prenatally exposed to DES and they are just now approaching the age at which breast cancer becomes more common." (http://www.aacr.org, 7 August 2006).

The findings prompted Palmer to warn affected women to avoid using hormone supplements in later life. Heather Feigelson, senior epidemiologist at the American Cancer Society, also advises caution: "Most of the women in this cohort are now in their early 40 s and 50 s, and we do not know whether their breast cancer risk will continue to rise." (http://www.cancer.org, 7 August 2006).

Although DES was withdrawn in 1971, the recent findings underscore the drug's legacy of concern, as expressed by Jane Kevan of the UK campaign group DES Action: “It's another thing to worry about. Where is it all going to end?" (http://news. scotsman.com, 8 August 2006).

Edward Wawrzynczak

\section{TUMORIGENESIS}

\section{Decidedly different}

The aberrant expression of oncogenes induces tumour development, but it also triggers apoptosis or cell-cycle arrest. So, cooperating mutations are needed to overcome these barriers, but which is more cooperative: loss of apoptosis or loss of cell-cycle arrest?

Previous work from Gerard Evan's group has shown that the activation of a regulatable form of the MYC protein (MYC-ER ${ }^{\text {TAM }}$ ) in the insulinsecreting $\beta$-islet cells ( $\beta$-cells) of the pancreas (plns-Myc-ER ${ }^{\text {TAM }}$ mice) results in the apoptosis of these cells. Inhibiting MYC-induced apoptosis through the expression of the anti-apoptotic protein $B C L-X_{L}$ results in an increase in $\beta$-cell numbers.

Other mutations, such as the loss of the tumour suppressors ARF or p53 also cooperate with MYC, but do they also work by suppressing MYC-induced apoptosis? Evan's group crossed the plns-Myc-ER ${ }^{\text {TAM }}$ mice with $\mathrm{Arf}^{-/-}$or Trp53 $3^{-/-}$ mice. Surprisingly, the $\beta$-cells of the plns-Myc$E R^{\text {TAM }} / \mathrm{Arf}^{-/-}$mice had higher rates of MYCinduced apoptosis than plns-Myc-ER $R^{\text {TAM }}$ mice, and higher rates of proliferation than the $\beta$-cells that expressed both MYC-ER ${ }^{\text {TAM }}$ and BCL-X. However, this increased proliferation in the plns-Myc-ER TAM $^{\text {TArf }}{ }^{-1-}$ mice did not cause an expansion of the $\beta$-cell population owing to the high levels of apoptosis. $\beta$-cells from the plns$M y c-E R^{T A M} / \operatorname{Trp} 53^{-/-}$mice showed an increased rate of proliferation similar to the plns-Myc$E R^{\text {TAM }} / \mathrm{Arf}^{-1-}$ mice, but rates of apoptosis were lower in this case than in plns-Myc-ER ${ }^{\text {TAM }}$ mice.

The aberrant expression of MYC normally induces the expression of ARF, which leads to the stabilization of $\mathrm{p} 53$, expression of the antiproliferative, cyclin-dependent-kinase inhibitor p21 and the induction of apoptosis. The expression of p21 does not occur in the Arf or Trp53 knockout cells, which leaves MYCinduced proliferation unchecked, but apoptosis is not affected. These data imply several important points. First, that ARF limits the oncogenicity of MYC by inhibiting proliferation; second, that this is p53 dependent; and third, that ARF does not limit the oncogenicity of MYC by inducing apoptosis. The lack of p21 expression also explains the increased proliferation in the plns-Myc-ER ${ }^{\text {TAM }} / \operatorname{Tr} p 53^{-1-}$ mice. The authors suggest that MYC-induced apoptosis is partially inhibited in the p53deficient mice because $\mathrm{p} 53$ is directly involved in inducing apoptosis by upregulating the expression of pro-apoptotic genes such as Puma, Noxa and BAX.

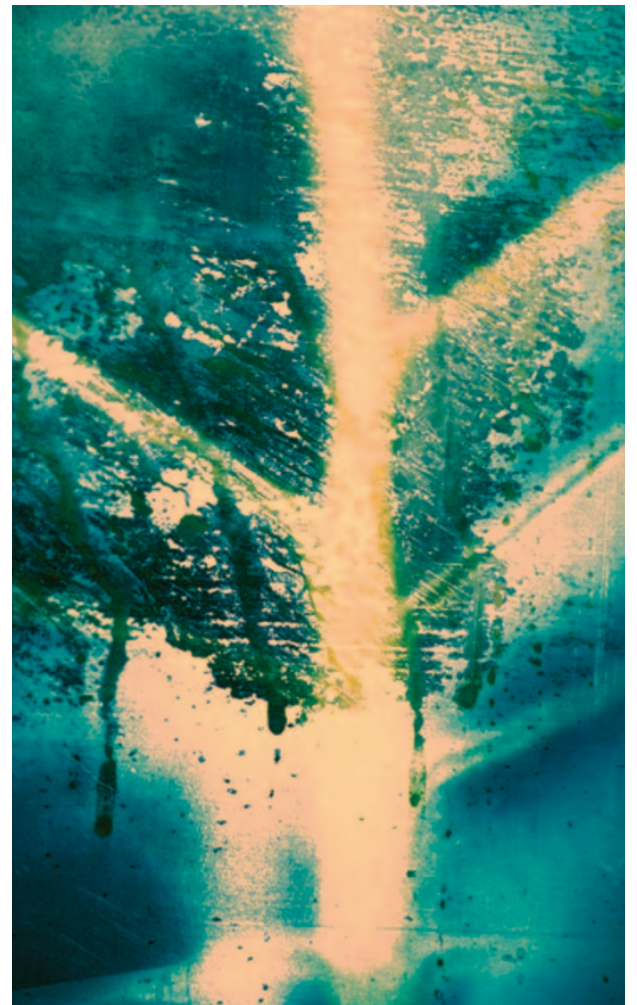

As ARF loss cooperates with MYC by increasing proliferation, and $\mathrm{BCL}-\mathrm{X}_{\mathrm{L}}$ cooperates by suppressing MYC-induced apoptosis, will the combination of ARF loss and BCL- $X_{L}$ expression further increase the oncogenicity of MYC? When MYC was activated, plns-Myc-ERTAM/RIP7-Bcl-x/ $A r f^{-1-}$ mice rapidly developed invasive tumours in which apoptosis was suppressed. Because MYCinduced apoptosis is partially suppressed in the p53-deficient background, plns-Myc-ER $R^{\text {TAM }} / \operatorname{Trp} 53^{-/ /}$ mice also develop invasive tumours. However, the tumours remain histologically different - there is no apoptosis in the tumours of plns-Myc-ER ${ }^{\text {TAM } /}$ RIP7-Bcl- $x_{L} / A^{\prime-1-}$ mice, but apoptosis persists in

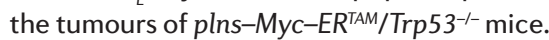

These results increase our understanding of how these pathways synergize in mouse models, but the relative importance of suppression of apoptosis and further increased proliferation is still unclear in the evolution and protracted development of human tumours.

Nicola McCarthy

ORIGINAL RESEARCH PAPER Finch, A. et al. Bcl-x gain of function and $p 19^{A R F}$ loss of function cooperate oncogenically with Myc in vivo by distinct mechanisms. Cancer Cell 10, 113-130 (2006) FURTHER READING Pelengaris, S., Khan, M. \& Evan, G. c-MYC: more than just a matter of life and death. Nature Rev. Cancer 2 764-776 (2002) 


\section{Transmission possible}

Healthy humans cannot 'catch' cancer through direct contact with tumours, but dogs can. Claudio Murgia and colleagues show that in canine transmissible venereal tumour (CTVT) it is the tumour cell that is the transmissible agent.

CTVT is a histiocytic tumour that is transmitted between dogs primarily during coitus and was first characterized 130 years ago. It has long been thought that the CTVT cells are able to engraft onto new hosts, but other data have indicated the involvement of an oncogenic virus. The authors used molecular genetic markers as a means to resolve the issue of transmission and to investigate the origin of CTVT.

Matched tumours and normal tissues were used from dogs treated in Italy, India and Kenya, and these were compared with archive tumour tissue isolated from sources worldwide. CTVT cells have previously been shown to harbour a long interspersed nuclear element (LINE-1) inserted close to the MYC oncogene, and the authors confirmed that this is a characteristic of the CTVT cell and not an inherited predisposition in the dogs themselves. Analyses of polymorphisms within the canine major histocompatability complex (MHC) genes, genotyping of microsatellite markers and analyses of the polymorphic control region of mitochondrial DNA show that the tumour cells and their hosts are genetically distinct. Further analyses indicate that CTVT might have originated in wolves, and that the current tumour clone is between 250 and 2,500 years old.

Interestingly, the microsatellite data also indicate that although the tumour cells are aneuploid, their genome is surprisingly stable. This could be because CTVT cells express telomerase, which can protect against DNA damage resulting from short telomeres.

CTVT cells can engraft in many different breeds of dog, but dogs that have recovered (gone on to reject the tumour) are immune on tumour rechallenge, indicating that the immune response must be suppressed by the tumour when it first establishes in a new host. Indeed, CTVT cells secrete transforming growth factor- $\beta$, a known immunosuppresive cytokine, and analysis of fresh tumour material from one of the dogs indicated reduced MHC expression compared with control tissue. So, CTVT seems to have adapted to initially evade the host immune response.

The expression of MHC genes is often reduced in tumours, so it is unclear why parasitic tumours have not emerged more frequently, over and above those identified in a colony of Syrian hamsters and the recent transmissible tumour outbreak in Tasmanian devils. The lack of genetic diversity in devils and hamsters might help to explain this in these species, but the confirmation that CTVT is also a parasitic tumour that has been stably aneuploid over many generations with a wide host range challenges many of our ideas about tumour development.

Nicola McCarthy

\section{ORIGINAL RESEARCH PAPER Murgia, C. et al.}

Clonal origin and evolution of a transmissible cancer. Cell 126, 477-487 (2006)

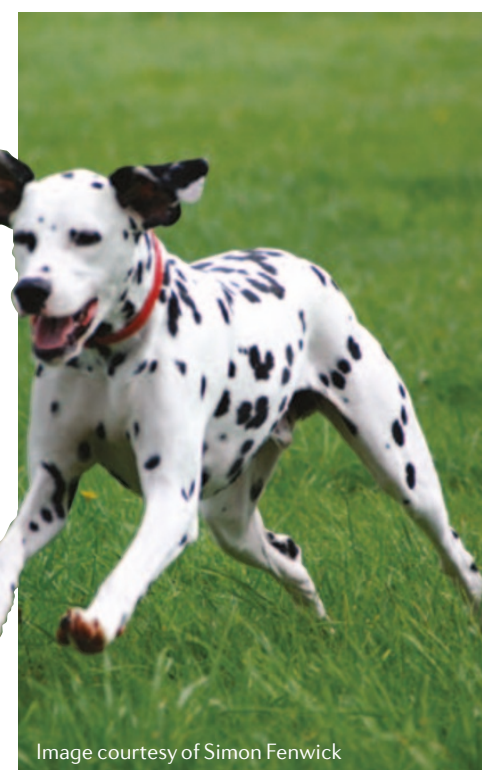

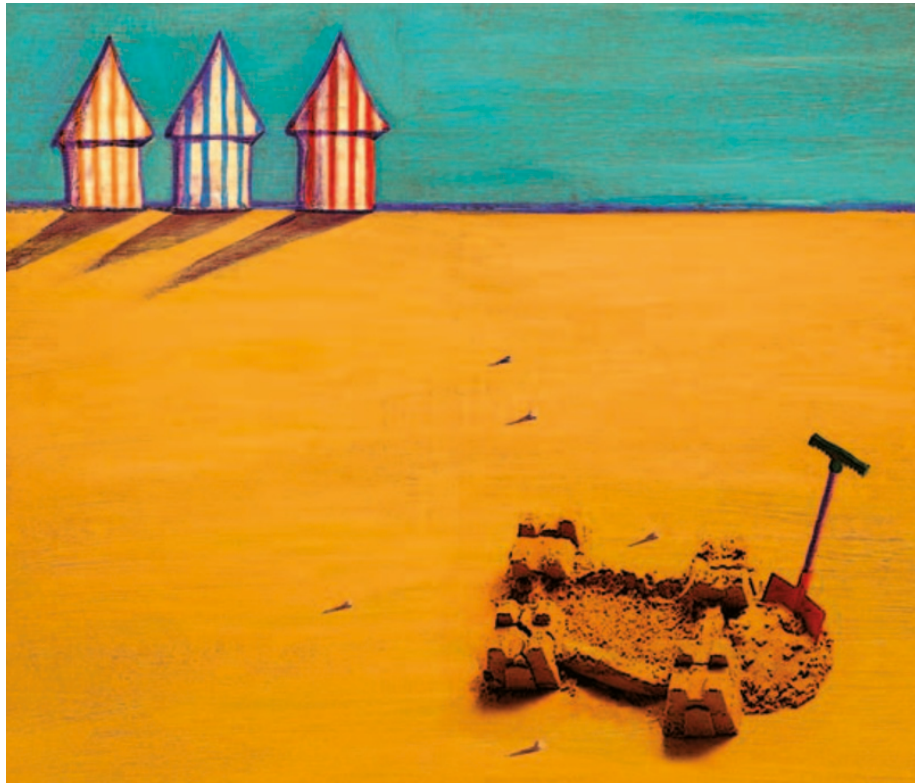

ANTICANCER AGENTS

\section{Digging in}

There are many essential processes that are carried out in a cell, and some of these rely on the cofactor NAD+. Javed Khan and colleagues have identified a means of specifically inhibiting $\mathrm{NAD}^{+}$production, a process known to induce tumour cell death.

Many of the biochemical processes that require $\mathrm{NAD}^{+}$, such as the repair of DNA strand breaks involving the enzyme poly (ADP-ribose) polymerase, lead to a decrease of NAD ${ }^{+}$ molecules. These are replenished by a salvage pathway that involves the enzyme nicotinamide phosphoribosyltransferase (NMPRTase). Inhibitors of NMPRTase have been known for some time, but it has not been clear how they function. So Khan and colleagues crystallized mouse and human NMPRTase with substrate, with an inhibitor and with neither, and solved their structures.

They found that NMPRTase is a dimer, and that there is a 'tunnel' at the interface between the two monomers, with the active site at one end of the tunnel. The co-crystal structure of NMPRTase with the inhibitor FK866 showed that FK866 binds tightly within the tunnel and prevents access to the active site. The shape of the tunnel restrains the types of chemicals, and therefore inhibitors, that can bind, and the presence of the tunnel in the NMPRTase, but not the related enzymes NAPRTase or QAPRTase, should ensure specificity.

FK866 will induce apoptosis in tumour cells that have increased levels of NMPRTase activity, which indicates that NMPRTase is a target worth pursuing for cancer therapy.

Nicola McCarthy

ORIGINAL RESEARCH PAPER Khan, J.A., Tao, X. \& Tong, L. Molecular basis for the inhibition of human NMPRTase, a novel target for anticancer agents. Nature Struct. Mol. Biol. 13, 582-588 (2006) 


\section{Anger management}

Metastatic tumour cells share many characteristics with embryonic progenitor cells, so investigating the behaviour of metastatic cells during embryogenesis in models

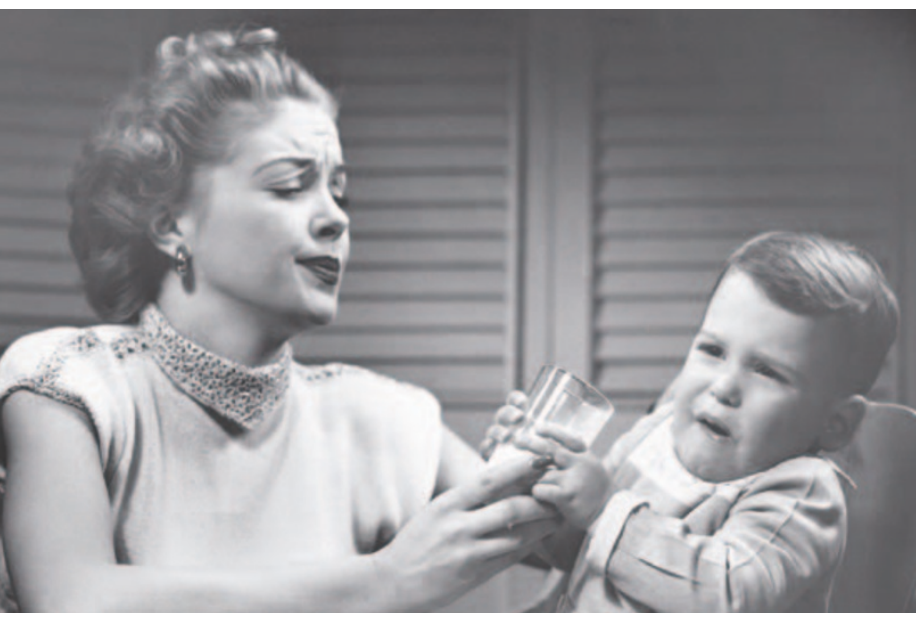

TUMORIGENESIS

\section{Stress and cancer}

Chronic stress has been suggested to increase tumour growth, but the mechanism has remained unclear. Anil Sood and colleagues have now shown that $\beta$-adrenergic signalling mediates increased angiogenesis and tumour growth in a mouse model.

The authors used nude mice that had been inoculated with human ovarian carcinoma cells, and the mice were stressed by being immobilized for several hours a day. Stressed mice had a three-fourfold increase in the number of tumour nodules and tumour weight; they also had more metastases. These results were replicated using other tumour cell lines and another method of stressing the mice.

The stressed mice had larger adrenal glands and greater sympathetic nervous system activity than the controls, so the authors investigated whether the effect of stress on tumour growth is mediated by $\beta$-adrenergic receptors. An agonist such as zebrafish (Danio rerio) can reveal common functional aspects. Mary Hendrix and colleagues have found that aggressively metastatic melanoma cells can disrupt early embryonic tissue patterning because they secrete the morphogen Nodal. Furthermore, they show that the expression of Nodal in human melanomas correlates well with aggressive forms of the disease.

Hendrix and colleagues have previously shown that when injected into zebrafish embryos, metastatic melanoma cells can respond to many environmental cues and do not form tumours. To explore the biology of aggressive melanoma cells more comprehensively within the zebrafish system, the authors investigated whether human melanoma cells could affect early stages of zebrafish development.
The injection of aggressively metastatic melanoma cells resulted in the formation of an additional body axis. Although many signalling pathways, such as Wnt and transforming growth factor- $\beta$ (TGF $\beta$ ), can produce a similar effect, only a few factors can produce such well-formed axes. Nodal is one such factor, and the authors found that highly aggressive melanoma cells express Nodal, whereas less aggressive melanoma cells do not. In agreement with this, Hendrix and colleagues found that Nodal expression was absent in normal human skin samples, was only weakly expressed in primary melanomas, but was expressed in $60 \%$ of metastatic melanoma samples, as determined by immunostaining.

Nodal is a member of the TGF $\beta$ superfamily and binds the activinlike (Alk) family of kinase receptors. As Nodal is known to be involved in maintaining the pluripotent status (plasticity) of embryonic stem cells, the authors examined the expression of differentiation markers in the the authors screened other ovarian cancer cell lines to find ones that were negative for the gene that encodes this receptor. They identified two such lines and found that stress had no significant effect on nodule number or tumour size. They also confirmed that it was the $\beta_{2}$-adrenergic receptors expressed on the human tumour cells rather than normal mouse cells that were important, as the effect could be reversed with

\section{Stressed mice had a three- agonists had no effect. \\ To confirm that the effect of stress is mediated by $\beta_{2}$-adrenergic receptors,} fourfold increase in the number of tumour nodules and tumour weight for $\beta_{2}$-adrenergic receptor and a general $\beta$-adrenergic receptor agonist increased tumour nodule number and chronic stress. Moreover, a $\beta$-adre antagonist could reverse these effects and the effects of chronic stress itself. By contrast, $\beta_{1}$-adrenergic receptor 


\section{RESEARCH HIGHLIGHTS}

aggressive cells. The inhibition of Alk receptors (which reduces the expression of Nodal) or the expression of Nodal-targeted oligonucleotides increased the expression of melanocyte differentiation markers in the aggressive melanoma cells, indicating that Nodal expression impedes differentiation and promotes plasticity.

Importantly, inhibiting Nodal expression also suppressed the capacity of the aggressive melanoma cells to invade the extracellular matrix, to form colonies in soft agar and to form tumours when injected subcutaneously into nude mice.

These results indicate that the expression of Nodal might be a good diagnostic marker for disease progression in patients with melanoma, and that Nodal is a potential target for the treatment of aggressive disease.

Nicola McCarthy

ORIGINAL RESEARCH PAPER Topczewska, J. M. et al. Embryonic and tumorigenic pathways converge via Nodal signaling: role in melanoma aggressiveness. Nature Med. 30 July 2006 (doi:10.1038/nm1448)

RNA interference that was specific for the human mRNA, and therefore did not affect the host cells.

As stress and $\beta_{2}$-adrenergic receptor agonists led to increased angiogenesis, the authors looked at the expression of vascular endothelial growth factor (VEGF) and found that it was upregulated. By using activators and inhibitors of adenylyl cyclase and protein kinase $\mathrm{A}$ (PKA), they showed that $\beta_{2}$-adrenergic receptors increase VEGF expression through the cAMP-PKA pathway.

These results show one way in which stress increases tumour growth, though they do not rule out other mechanisms being involved in different circumstances. They also suggest that targeting angiogenesis by blocking $\beta_{2}$-adrenergic receptor signalling could be useful in ovarian cancer therapy.

Patrick Goymer

ORIGINAL RESEARCH PAPER Thaker, P. H. et al. Chronic stress promotes tumor growth and angiogenesis in a mouse model of ovarian carcinoma. Nature Med. 23 July 2006

(doi:10.1038/nm1447)
TARGETED THERAPIES

\section{The next move}

Mutations in the epidermal growth factor receptor (EGFR, also known as ERBB1) that result in resistance to the EGFR inhibitor gefitinib have prompted closer examination of EGFR in patients with non-small-cell lung cancer (NSCLC). Changes in the dimerization partners of EGFR, such as ERBB2 (also known as HER2), or triggering alternative ErbB signalling pathways, such as ERBB3 (also known as HER3), have a role in gefitinib resistance. Two groups have investigated the next move to circumvent this resistance Carlos Arteaga and colleagues show that patients with intragenic mutations in the kinase domain of ERBB2 are sensitive to ERBB2 inhibitors, and Bin-Bing Zhou and colleagues show that the inhibition of an upstream activator of the ligand for ERBB3, ADAM17, causes the death of certain gefitinib-resistant NSCLC cells.

About $2 \%$ of patients with NSCLC have intragenic mutations in ERBB2, which is a preferred dimerization partner of EGFR and leads to the activation of ErbB signalling pathways. But how does this mutation affect tumour cells? When Arteaga and colleagues transfected human immortalized bronchial or mammary epithelial cell lines with the ERBB2 mutant (encoding ERBB2 ${ }^{\text {YVMA }}$ ) or wild-type ERBB2, only cells with the mutant receptor formed tumours in nude mice. Cells transfected with the gene encoding ERBB2 ${ }^{\mathrm{WT}}$ or ERBB2 ${ }^{\mathrm{YVMA}}$ and treated with a bivalent ligand to induce receptor homodimerization showed that ERBB2 ${ }^{\text {YVMA }}$ phosphorylation - which was high even in the absence of ligand - was more inducible than ERBB2 ${ }^{\mathrm{WT}}$. In addition, basal levels of phosphorylation and, therefore, activation of the signalling molecules EGFR, SRC, AKT and MAPK were markedly higher in ERBB2 ${ }^{\text {YVMA }}$ cells. Treatment with gefitinib efficiently blocked ligand-induced target phosphorylation in the cells that expressed ERBB2 ${ }^{\text {WT }}$, probably through interference with EGFR-ERBB2 crosstalk. However, the inhibition of EGFR by gefitinib had no affect on the constitutively active ERBB2 mutant or on its ability to engage with and activate ErbB signal transducers.

So, could this resistance be circumvented by inhibiting ERBB2 rather than EGFR? Arteaga and colleagues found that the ERBB2 antibody trastuzumab inhibited the growth and invasion of cells that expressed the ERBB2 mutant. In addition, treatment with the EGFR and ERBB2 dual inhibitors lapatinib or $\mathrm{Cl}-1033$ inhibited growth and invasion of both wild-type and mutant cells. The combination of lapatinib and trastuzumab was more potent than either drug alone in suppressing

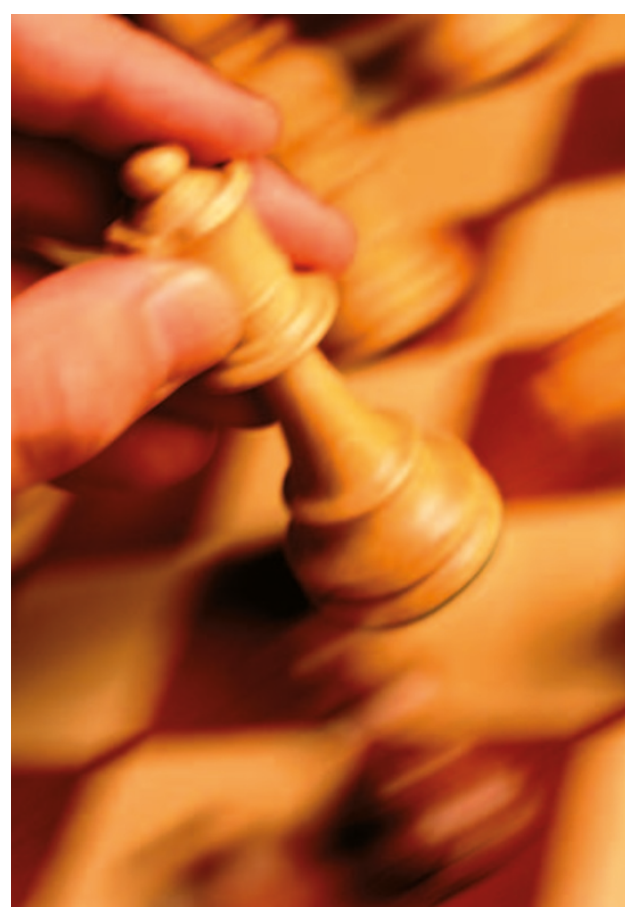

the growth of mutant cells. Knockdown of mutant ERBB2 also resulted in the inhibition of cell proliferation and cell death, and restored sensitivity to gefitinib in lung cancer cells.

Zhou et al. examined the activation of an alternative ErbB pathway, ERBB3 (also known as HER3), as a possible mechanism of resistance to gefitinib. The only known ligand for ERBB3 is heregulin, and this needs be to proteolytically cleaved to become active. ADAM17 is a metalloprotease that is thought to mediate this cleavage. The authors found that many primary tumours from NSCLC patients that they examined had activated ERBB3, co-expressed heregulin and ERBB3, and had increased expression of ADAM17. Heregulin-induced ERRB3 activation in NSCLC cell lines caused resistance to gefitinib. RNA interference of ADAM17 or treatment with a small-molecule inhibitor of ADAM 17, INCB3619, prevented the cleavage of heregulin and abrogated heregulin-dependent ERBB3 signalling in A549 NSCLC cells. Furthermore, the INCB3619treated $A 549$ cells were then resensitized to treatment with gefitinib.

So, both these reports have increased our understanding of the ErbB signalling pathways and the approaches explored hold promise for overcoming resistance to gefitinib in NSCLC.

\section{Ezzie Hutchinson}

ORIGINAL RESEARCH PAPERS Wang, S. E. et al. ERBB2 kinase domain mutation results in constitutive phosphorylation and activation of ERBB2 and EGFR and resistance to EGFR tyrosine kinase inhibitors. Cancer Cell 10, 25-38 (2006) |Zhou, B.-B. S. et al. Targeting ADAM-mediated ligand cleavage to inhibit HER3 and EGFR pathways in non-small cell lung cancer. Cancer Cell 10, 39-50 (2006) 


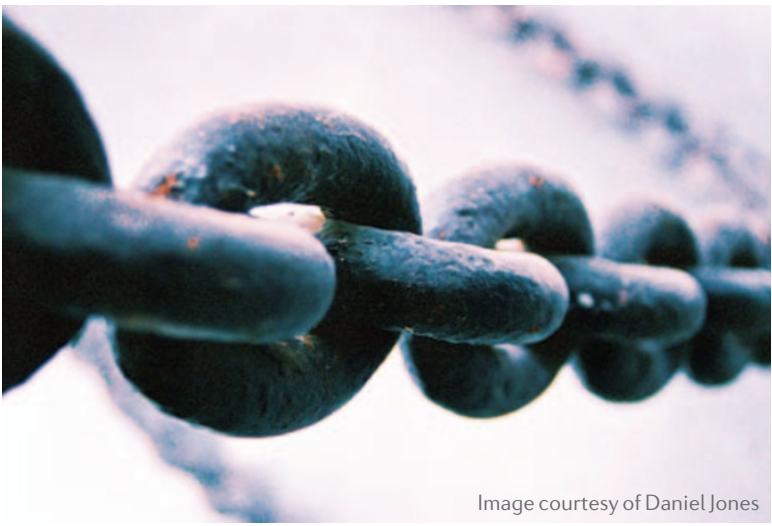

$\Rightarrow$ THERAPY

\section{Through the barricades}

The blood-brain barrier serves to protect the brain from bloodborne infections and toxic agents, making the treatment of brain metastasis with systemic agents particularly difficult. For the most part this has been overcome by injecting chemotherapy agents into the cerebrospinal fluid, but this approach is not ideal. With the advent of new targeted therapies, such as the monoclonal antibody trastuzumab (Herceptin) that effectively treats ERBB2-positive breast cancers, a need has arisen to deliver such agents to the brain to help eradicate brain metastasis. Manabu Kinoshita and colleagues have succeeded in getting trastuzumab to cross the blood-brain barrier in mice through the use of focused ultrasound.

Focused ultrasound, used at a high frequency, is able to concentrate acoustic energy on an area of tissue a few millimetres in diameter and is used to kill tumour tissue. The addition of an ultrasound contrast agent that produces bubbles results in transient changes in cell-membrane permeability and is known to disrupt the blood-brain barrier. The authors tested whether high frequency ultrasound could be used to transiently disrupt the blood-brain barrier so that trastuzumab could reach brain tissue in mice. Magnetic resonance imaging was used to guide the beam of focused ultrasound.

Initially Kinoshita and colleagues showed that this technique could disrupt the blood-brain barrier to enable the entrance of the vital dye trypan blue. They then injected trastuzumab systemically into 13 mice; 9 mice were given ultrasound with a contrast agent, the other 4 were not. Significantly more trastuzumab was detected in the brains of mice that were treated with ultrasound compared with those that were given the antibody alone. Importantly, even where there was evidence of significant disruption to the blood-brain barrier the underlying brain tissue was not damaged.

This technique might prove useful for the treatment of brain metastasis with antibody-based targeted agents.

Nicola McCarthy

ORIGINAL RESEARCH PAPER Kinoshita, M., McDannold, N., Jolesz, F. A. $\varepsilon$ Hynynen, K. Noninvasive localized delivery of Herceptin to the mouse brain by MRI-guided focused ultrasound-induced blood-brain barrier disruption. Proc. Natl Acad. Sci. USA 103, 11719-11723 (2006).

FURTHER READING Kennedy]. E. High-intensity focused ultrasound in the

treatment of solid tumours. Nature Rev. Cancer 5, 321-327 (2005)

\section{PHARMACODYNAMICS}

\section{A new model}

Kevin Hicks, William Wilson and colleagues have combined in vitro and in vivo data to produce a mathematical model that describes drug action as a function of the distance of the target from blood vessels. It can be used to predict anti-tumour efficacy in human tumour xenografts.

The plasma concentration of a therapeutic drug is often used as a surrogate for the concentration of the drug in the tumour. However, the disorganized nature of tumour vasculature means that drugs must diffuse over considerable distances to reach all their target cells. Poor penetration of a drug through the extravascular compartment of a tumour is therefore likely to result in therapy failure.
To better reflect this reality, the authors measured the tissue penetration properties of a hypoxia-targeted drug, tirapazamine (TPZ), that is specifically toxic to hypoxic tumour cells because it is reduced to a DNA-damaging free radical under hypoxic conditions. They measured the diffusion of TPZ using their in vitro $3 \mathrm{D}$ cell-culture model in which HT29 colon cancer cells are grown as multicellular layers. They also measured the plasma pharmacokinetics (PK; drug concentration over time) of TPZ in nude mice and the metabolism and cytotoxicity of TPZ in stirred, anoxic cultures of HT29 cells. The authors then used these data to model drug transport in a well-characterized microvascular network of a rat mammary carcinoma in which parameters such as oxygen

\section{Eph-ective tumour growth inhibition}

Although receptor tyrosine kinases typically activate oncogenic pathways, Elena Pasquale and colleagues have found that stimulating a member of the ephrin (Eph) receptor tyrosine kinase family inhibits breast tumour cell growth in mice.

Expression of the EPHB4 receptor is

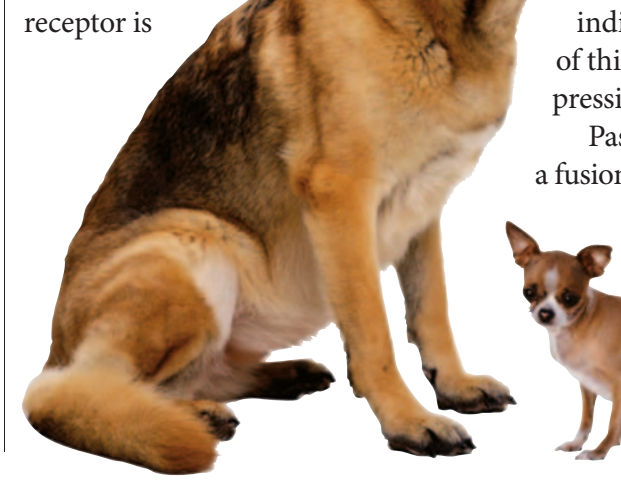

maintained in many cancers, including breast cancers, but expression of the EPHB4 ligand ephrin B2

(EFNB2) is lost, which prevents activation of the receptor and downstream signalling pathways. Conversely, both receptor and ligand are expressed in non-transformed human breast epithelial cells, which indicates the possible importance of this pathway in tumour suppression.

Pasquale and colleagues used fusion protein of EFNB2 and the Fc portion of human immunoglobulin G1. The stimulation of EPHB4 with EFNB2-Fc in a breast cancer xenograft model in nude mice 
transport and the direction and velocity of blood flow are known. Their resulting PK and pharmacodynamic (PD; effect of the drug) mathematical model predicted that the access of TPZ to the hypoxic regions of a tumour would be compromised by its diffusion and metabolic properties to the extent that the maximum number of hypoxic cells killed would be much less than if TPZ behaved in accordance with its plasma PK properties alone.

The authors validated the model by predicting and then measuring the efficacy of 15 TPZ analogues with different tumour-penetration properties. There was a strong correlation between model-predicted and actual numbers of cells killed in HT29 xenografts as determined by clonogenic assay. However, this correlation was lost if the model was based on plasma PK only.

Wilson and colleagues conclude that data from in vitro 3D tissue-culture models combined with PK and PD models that consider extravascular drug diffusion are useful for predicting the efficacy of drugs in tumour tissue. This might also reduce the numbers of animals required to test the initial efficacy of new chemotherapeutic drugs.

Nicola McCarthy

ORIGINAL RESEARCH PAPER Hicks, K. et al. Use of three-dimensional tissue cultures to model extravascular transport and predict in vivo activity of hypoxia-targeted anticancer drugs. J. Natl Cancer Inst. 16 July 2006 (doi:10.1093/jnci/djj306) FURTHER READING Drug penetration in solid tumours. Minchinton, A. I. \& Tannock, I. F. Nature Rev. Cancer 6, 583-592 (2006)

\section{IN BRIEF}

\section{$\Rightarrow$ ANGIOGENESIS}

Augmentation of tumour angiogenesis by a MYC-activated microRNA cluster

Dews, M. et al. Nature Genet. 30 July 2006 (doi: 10.1038/ng1855)

MYC expression in tumour cells increases tumour neovascularization. Dews et al. show that the transduction of mouse colonocytes that express KRAS with a MYC-encoding retrovirus stimulates angiogenesis and the concomitant downregulation of anti-angiogenic thrombospondin 1 (TSP1). Antisense inhibition of the mir-17-92 microRNA cluster, which is upregulated in the transformed colonocytes, partly restores TSP1 expression, and transduction with an miR-17-92-encoding retrovirus mimics the effect of MYC transduction, establishing a role for this microRNA in tumour angiogenesis.

\section{$\Rightarrow$ TUMOUR SUPPRESSORS}

\section{A CK2-dependent mechanism for degradation of the} PML tumour suppressor

Scaglione, P. P. et al. Cell 126, 269-283 (2006)

The loss of expression of PML in human cancers correlates with tumour progression. Scaglione et al. demonstrate that casein kinase 2 (CK2) phosphorylates the PML protein and promotes its ubiquitin-mediated degradation. The tumour-suppressor function of PML is increased in cells that express PML mutants that are resistant to CK2 phosphorylation or CK2 inhibition. This regulatory mechanism explains the inverse correlation between CK2 kinase activity and PML protein levels in human lung cancer cells.

significantly inhibited tumour growth. Furthermore, the mice treated with EFNB2-Fc did not lose weight, which indicates that the treatment is relatively non-toxic.

Which signalling pathways are responsible for the negative effect of EPHB4 on tumour growth? Using three breast cancer cell lines, the authors showed that EFNB2-Fc stimulated tyrosine phosphorylation of CRK, which functions downstream of active EPHB4. CRK was also highly phosphorylated in the tumour xenografts that were treated with EFNB2-Fc. The phosphorylation of CRK on tyrosine 221 induces a conformational change that blocks the ability of CRK to function as an adaptor protein. Phosphospecific antibodies and the mutation of this site confirmed that this is the primary tyrosine residue in CRK that is phosphorylated downstream of active EPHB4. The phosphorylation of tyrosine 221 is known to be mediated by the kinase $\mathrm{ABL}$, and the treatment of cells with the ABL inhibitor imatinib or $A B L$ small interfering RNA blocked CRK phosphorylation in response to EFNB2-Fc stimulation.
CRK binds the scaffolding protein CAS (also known as BCAR1), linking it to pathways that regulate cell survival. Indeed, the authors found that the treatment of cells with EFNB2-Fc inhibited the interaction of CAS with CRK in breast cancer cells. The examination of a breast cancer cell line grown in culture dishes and as 3D spheroids showed that the consequence of EPHB4 activation was to reduce cell numbers both by inducing apoptosis and inhibiting DNA synthesis, and this required the $\mathrm{ABL}-\mathrm{CRK}$ signalling pathway. ABL activation was also crucial for inhibiting the growth of tumour xenografts.

The negative effect of EPHB4 activation on tumour growth and the fact that many breast tumours retain expression of EPHB4 indicate that stimulating this receptor might be a valid therapeutic strategy for breast cancer.

Sarah Seton-Rogers

ORIGINAL RESEARCH PAPER Noren, N. K. et al. The EphB4 receptor suppresses breast cancer cell tumorigenicity through an Abl-Crk pathway. Nature Cell Biol. 8, 815-825 (2006)

\section{$\Rightarrow$ METASTASIS}

Interaction of KAI 1 on tumour cells with DARC on vascular endothelium leads to metastasis suppression

Bandyopadhyay, S. et al. Nature Med. 12, 933-938 (2006)

KAl1 is a cell-surface protein associated with suppressing metastasis in various human tumours. To investigate how KAl1 achieves this, the authors used a yeast two-hybrid screen to identify KAl1-interacting proteins. Duffy antigen receptor for chemokines (DARC) is one such protein and is expressed on endothelial cells. The authors found that KAl1-expressing tumour cells that enter a blood vessel (intravasate) bind DARC and undergo senescence. Furthermore, cells that express KAl1 were able to metastasize in DARC-knockout mice, showing that the KAl1-DARC metastasis-suppression pathway is activated once tumour cells have intravasated.

\section{$\Rightarrow$ CELL DEATH}

DRAM, a p53-induced modulator of autophagy, is critical for apoptosis

Crighton, D. et al. Cell 126, 121-134 (2006)

The transcription factor and tumour suppressor $\mathrm{p} 53$ can induce cell-cycle arrest and apoptosis. Kevin Ryan and colleagues have identified a new p53 gene target, damageregulated autophagy modulator (DRAM), which induces autophagy and contributes to p53-mediated apoptosis. The expression of DRAM mRNA is decreased in various squamous-cell tumours with functional p53 expression, indicating that the modulation of both apoptosis and autophagy might be important for p53-mediated tumour suppression. 